\title{
Origin of the second coherent peak in the dynamical structure factor of an asymmetric spin-ladder
}

\author{
P. N. Bibikov and M. I. Vyazovsky \\ V. A. Fock Institute of Physics, Sankt-Petersburg State University
}

September 11, 2018

\begin{abstract}
Appearance of the second coherent peak in the dynamical structure factor of an asymmetric spin ladder is suggested. The general arguments are confirmed by the first order (with respect to the asymmetry) calculation for a spin ladder with singlet-rung ground state. Basing on this result a new interpretation is proposed for the inelastic neutron scattering data in the spin gap compound $\mathrm{CuHpCl}$ for which some of the corresponding interaction constants are estimated.
\end{abstract}

\section{Introduction}

Inelastic neutron scattering is an effective method for analysis of low-energy excitations in low-dimensional spin systems [1]. The dynamical structure factor (DSF) obtained from this experiment produces an essential information about the low-energy spectrum. Sharp peaks of the DSF line shape correspond to coherent modes while broad bands originate from incoherent excitation continuums.

Theoretical study of a spin ladder DSF was developed in the papers [2], [3]. A strong antiferromagnetic rung coupling corresponds to the DSF with a single coherent-mode peak [2], while for a weak coupling the line shape has only an incoherent background [3]. The models studied in [2], [3] are symmetric under exchange of the legs because their couplings 
along both legs are equal to each other and the same is true for the couplings along both diagonals. Such requirement fails for an asymmetric spin ladder.

The compound $\mathrm{Cu}_{2}\left(\mathrm{C}_{5} \mathrm{H}_{12} \mathrm{~N}_{2}\right)_{2} \mathrm{Cl}_{4}(\mathrm{CuHpCl})$ was first interpreted as an asymmetric spin ladder [4] (with non equal couplings along diagonals). However neutron scattering [5], [6] revealed two coherent peaks in the DSF line shape for $\mathrm{CuHpCl}$. Since this type of behavior does not agree with the results of [2], [3] (obtained for the symmetric case!) it was suggested in [6] that the magnetic structure of $\mathrm{CuHpCl}$ is inconsistent with the spin ladder model.

In this paper we show the principal difference between excitation spectrums of symmetric and asymmetric spin ladders and suggest argumentation confirming the existence of the second coherent peak in DSF of a asymmetric spin ladder. As an example we calculate the DSF for a weakly asymmetric spin ladder with singlet-rung ground state and produce an evidence for the second coherent peak.

\section{Hamiltonian for an asymmetric spin ladder}

The general Hamiltonian of an asymmetric spin-ladder has the following form,

$$
\hat{H}=\hat{H}^{s y m m}+\hat{H}^{a s y m m}
$$

where $\hat{H}^{\text {symm }}=\sum_{n} H_{n, n+1}^{\text {symm }}$ and $\hat{H}^{\text {asymm }}=\sum_{n} H_{n, n+1}^{a s y m m}$. The local Hamiltonian densities are given by the following expressions:

$$
H_{n, n+1}^{\text {symm }}=H_{n, n+1}^{\text {rung }}+H_{n, n+1}^{\text {leg }}+H_{n, n+1}^{\text {frust }}+H_{n, n+1}^{c y c} .
$$

where

$$
\begin{aligned}
H_{n, n+1}^{\text {rung }} & =J_{\perp} \mathbf{S}_{1, n} \cdot \mathbf{S}_{2, n}, \\
H_{n, n+1}^{\text {leg }} & =J_{\|}^{\text {symm }}\left(\mathbf{S}_{1, n} \cdot \mathbf{S}_{1, n+1}+\mathbf{S}_{2, n} \cdot \mathbf{S}_{2, n+1}\right), \\
H_{n, n+1}^{\text {frust }} & =J_{\text {frust }}^{\text {symm }}\left(\mathbf{S}_{1, n} \cdot \mathbf{S}_{2, n+1}+\mathbf{S}_{2, n} \cdot \mathbf{S}_{1, n+1}\right), \\
H_{n, n+1}^{\text {cyc }} & =J_{c}\left(\left(\mathbf{S}_{1, n} \cdot \mathbf{S}_{1, n+1}\right)\left(\mathbf{S}_{2, n} \cdot \mathbf{S}_{2, n+1}\right)+\left(\mathbf{S}_{1, n} \cdot \mathbf{S}_{2, n}\right)\left(\mathbf{S}_{1, n+1} \cdot \mathbf{S}_{2, n+1}\right)\right. \\
& \left.-\left(\mathbf{S}_{1, n} \cdot \mathbf{S}_{2, n+1}\right)\left(\mathbf{S}_{2, n} \cdot \mathbf{S}_{1, n+1}\right)\right),
\end{aligned}
$$

and

$$
H_{n, n+1}^{\text {asymm }}=J_{\|}^{a s y m m}\left(\mathbf{S}_{1, n} \cdot \mathbf{S}_{1, n+1}-\mathbf{S}_{2, n} \cdot \mathbf{S}_{2, n+1}\right)+J_{\text {frust }}^{\text {asymm }}\left(\mathbf{S}_{1, n} \cdot \mathbf{S}_{2, n+1}-\mathbf{S}_{2, n} \cdot \mathbf{S}_{1, n+1}\right) .
$$




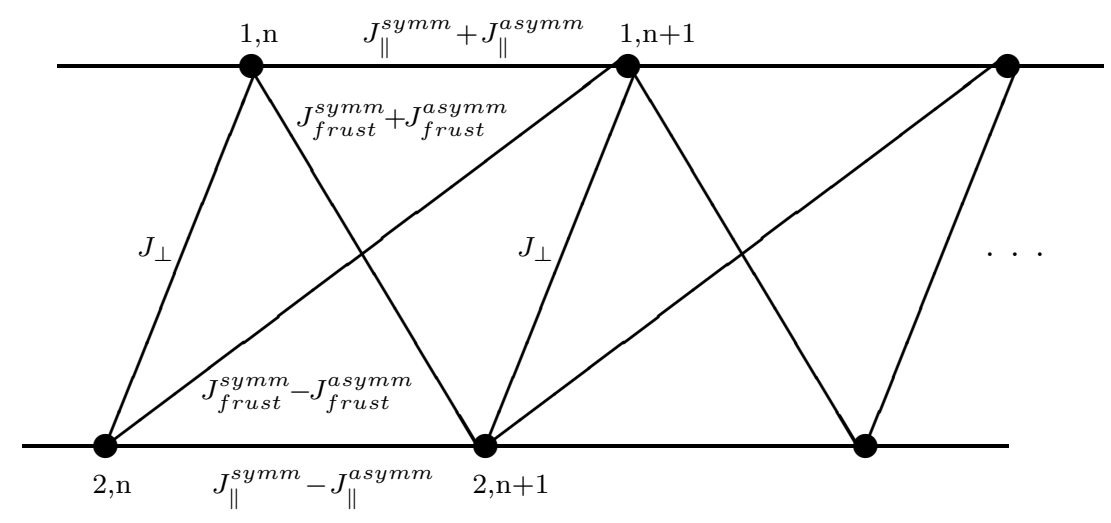

Figure 1: Schematic of the magnetic structure of an asymmetric spin ladder.

This structure is schematically represented on the Fig.1.

It is convenient to extract from the general $\hat{H}^{\text {symm }}$ the "singlet-rung" part $\hat{H}^{s-r}$ commuting with the following operator:

$$
\hat{Q}=\sum_{n} Q_{n}
$$

where $Q_{n}=\frac{1}{2}\left(\mathbf{S}_{1, n}+\mathbf{S}_{2, n}\right)^{2}$. The commutativity condition

$$
\left[\hat{H}^{s-r}, \hat{Q}\right]=0
$$

or in equivalent form

$$
\left[H_{n, n+1}^{s-r}, Q_{n}+Q_{n+1}\right]=0,
$$

results to the following restriction on the interaction constants for $\hat{H}^{s-r}$

$$
J_{\text {frust }}^{s-r}=J_{\|}^{s-r}-\frac{1}{2} J_{c}^{s-r} .
$$

According to (8) the Hilbert space for $\hat{H}^{s-r}$ splits on the infinite set of eigenspaces corresponding to different eigenvalues of $\hat{Q}[7],[8]$ :

$$
\mathcal{H}=\sum_{m=0}^{\infty} \mathcal{H}^{m},\left.\quad \hat{Q}\right|_{\mathcal{H}^{m}}=m .
$$

The one-dimensional subspace $\mathcal{H}^{0}$ is generated by the single vector

$$
|0\rangle=\prod_{n}|0\rangle_{n}
$$

where $|0\rangle_{n}$ is the $n$-th rung singlet. The following restrictions:

$$
J_{\perp}^{s-r}>2 J_{\|}^{s-r}, \quad J_{\perp}^{s-r}>\frac{5}{2} J_{c}^{s-r}, \quad J_{\perp}^{s-r}+J_{\|}^{s-r}>\frac{3}{4} J_{c}^{s-r},
$$


guarantee that the state $(10)$ is the (singlet-rung) ground state for $\hat{H}^{s-r}$. The operator $\hat{Q}$ has a sense of the magnon number [8] associated with $\hat{H}^{s-r}$.

Decomposition

$$
\hat{H}^{s y m m}=\hat{H}^{s-r}+\Delta \hat{H}^{s y m m} .
$$

will be correct only if we put some additional restrictions on the interaction constants of $\Delta \hat{H}^{\text {symm }}$. Postulating them in the form

$$
\Delta J_{\perp}^{\text {symm }}=\Delta J_{c}^{\text {symm }}=0, \quad \Delta J_{\|}^{\text {symm }}=-\Delta J_{\text {frust }}^{\text {symm }} .
$$

we guarantee the uniqueness of the decomposition (12). Moreover under (13) and (4) the local exchange relations between $\Delta \hat{H}^{\text {symm }}, \hat{H}^{\text {asymm }}$ and $\hat{Q}$ have the following form:

$$
\begin{aligned}
\left\{\Delta H_{n, n+1}^{\text {symm }}, Q_{n}+Q_{n+1}\right\} & =2 \Delta H_{n, n+1}^{\text {symm }}, \\
\left\{H_{n, n+1}^{a s y m m}, Q_{n}+Q_{n+1}\right\} & =3 H_{n, n+1}^{a s y m m},
\end{aligned}
$$

(where $\{$,$\} means anti commutator).$

As it follows from (14) the term $\Delta \hat{H}^{\text {symm }}$ does not mix even and odd components in (9). Therefore the Hilbert space $\mathcal{H}$ splits on two invariant subspaces, of the operator $\hat{H}^{\text {symm }}$

$$
\mathcal{H}=\mathcal{H}^{\text {even }}+\mathcal{H}^{\text {odd }}, \quad \mathcal{H}^{\text {even }}=\sum_{m=0}^{\infty} \mathcal{H}^{2 m}, \quad \mathcal{H}^{\text {odd }}=\sum_{m=0}^{\infty} \mathcal{H}^{2 m+1} .
$$

From (15) follows that $\hat{H}^{\text {asymm }}$ mixes $\mathcal{H}^{\text {even }}$ and $\mathcal{H}^{\text {odd }}$, however on the sector $\mathcal{H}^{0}$ its action is trivial. Really according to (15) $H_{n, n+1}^{a s y m m}|0\rangle_{n}|0\rangle_{n+1}$ have to lie in the sector with $Q_{n}+Q_{n+1}=3$ that is impossible because the operator $Q_{n}$ has only eigenvalues 0 and 1. So we have

$$
\hat{H}^{\text {asymm }}|0\rangle=0
$$

More detailed analysis of the $16 \times 16$ matrix $H^{\text {asymm }}$ (which represent the action of $H_{n, n+1}^{a s y m m}$ on the product of $n$-th and $n+1$-rungs) shows that it has only three (degenerative) eigenvalues: 0 , and $\pm \sqrt{\left.\left(J_{\|}^{\text {asymm }}\right)^{2}+J_{\text {frust }}^{\text {asymm }}\right)^{2}}$. Therefore for small $J_{\|}^{\text {asymm }}$ and $J_{\text {frust }}^{\text {asymm }}$ the state $|0\rangle$ remains to be the ground state for $\hat{H}^{s-r}+\hat{H}^{a s y m m}$.

Now we may suggest the following interpretation for the appearance of the second coherent mode in the DSF line shape of an asymmetric spin ladder. It is known [7][9] that in the strong rung-coupling regime an excitation spectrum of a symmetric spin ladder has coherent modes of two types, the one-magnon triplet state lying in $\mathcal{H}^{\text {odd }}$ and three bound two-magnon states (with total spin $0,1,2$ ) lying in $\mathcal{H}^{\text {even }}$. The ground state 
also lies in $\mathcal{H}^{\text {even }}$. In the Born approximation a scattering neutron creates a new state by flipping a single elementary spin. It is a principal fact that the excited state lies in $\mathcal{H}^{\text {odd }}$. By this reason in the symmetric case only the subspace $\mathcal{H}^{\text {even }}$ excites during the the scattering process. However even a little asymmetry results to excitations from $\mathcal{H}^{\text {even }}$ and in particular the bound two-magnon mode with total spin 1 which is respective for the appearance of the second coherent peak in the DSF.

In the next sections we shall confirm our arguments by studying the simplest model for which $\Delta \hat{H}^{\text {symm }}=0$ and the ground state exactly has the form (10).

\section{One and two-magnon states for $\hat{H}^{s-r}$}

The eigenstates of $\hat{H}^{s-r}$ in the sectors with $\hat{Q}=1$ and $\hat{Q}=2$ may be obtained exactly [7],[8]. From now we shall concern on this special model omitting the upper indexes " $s-r$ " or "symm" in notation of interaction constant $J_{c}, J_{\|}$and $J_{\text {frust }}$. In other words we shall study the model (2)-(3) with additional restrictions (8) and (11) on $J_{\perp}, J_{\|}, J_{\text {frust }}$ and $J_{c}$.

According to the following formulas:

$$
\begin{aligned}
H_{n, n+1}^{s-r}|0\rangle_{n}|1\rangle_{n+1}^{\alpha} & =\left(\frac{1}{2} J_{\perp}-\frac{3}{4} J_{c}\right)|0\rangle_{n}|1\rangle_{n+1}^{\alpha}+\frac{J_{c}}{2}|1\rangle_{n}^{\alpha}|0\rangle_{n+1}, \\
H_{n, n+1}^{s-r}|1\rangle_{n}^{\alpha}|0\rangle_{n+1} & =\left(\frac{1}{2} J_{\perp}-\frac{3}{4} J_{c}\right)|1\rangle_{n}^{\alpha}|0\rangle_{n+1}+\frac{J_{c}}{2}|0\rangle_{n}|1\rangle_{n+1}^{\alpha}, \\
H_{n, n+1}^{s-r} \varepsilon_{\alpha \beta \gamma}|1\rangle_{n}^{\beta}|1\rangle_{n+1}^{\gamma} & =\left(J_{\perp}-J_{\|}-J_{c} / 4\right) \varepsilon_{\alpha \beta \gamma}|1\rangle_{n}^{\beta}|1\rangle_{n+1}^{\gamma},
\end{aligned}
$$

where $\alpha, \beta, \gamma=1,2,3$ and $|1\rangle_{n}^{\alpha}$, is the triplet associated with a $n$-th rung:

$$
|1\rangle_{n}^{\alpha}=\left(\mathbf{S}_{1, n}^{\alpha}-\mathbf{S}_{2, n}^{\alpha}\right)|0\rangle, \quad\left(\mathbf{S}_{1, n}^{\alpha}+\mathbf{S}_{2, n}^{\alpha}\right)|1\rangle_{n}^{\beta}=i \varepsilon_{\alpha \beta \gamma}|1\rangle_{n}^{\gamma},
$$

one- and (spin-1) two-magnon states for $\hat{H}^{s-r}$ have the following form [7],[8]:

$$
\begin{aligned}
\mid k, \text { magn }\rangle_{0}^{\alpha} & =\frac{1}{\sqrt{N}} \sum_{n} e^{i k n} \ldots|0\rangle_{n-1}|1\rangle_{n}^{\alpha}|0\rangle_{n+1} \ldots \\
\left.\mid k_{1}, k_{2}, \text { scatt }\right\rangle_{0}^{\alpha} & =Z_{\text {scatt }}^{-1}\left(k_{1}, k_{2}\right) \sum_{m=-\infty}^{\infty} \sum_{n=m+1}^{\infty} \varepsilon_{\alpha \beta \gamma} a^{\text {scatt }}\left(m, n ; k_{1}, k_{2}\right) \ldots|1\rangle_{m}^{\beta} \ldots|1\rangle_{n}^{\gamma} \ldots \\
\mid k, \text { bound }\rangle_{0}^{\alpha} & =Z_{\text {bound }}^{-1}(k) \sum_{m=-\infty}^{\infty} \sum_{n=m+1}^{\infty} \varepsilon_{\alpha \beta \gamma} a^{\text {bound }}(m, n ; k) \ldots|1\rangle_{m}^{\beta} \ldots|1\rangle_{n}^{\gamma} \ldots
\end{aligned}
$$

where

$$
\begin{aligned}
a^{\text {scatt }}\left(m, n ; k_{1}, k_{2}\right) & =C_{12} \mathrm{e}^{i\left(k_{1} m+k_{2} n\right)}-C_{21} \mathrm{e}^{i\left(k_{2} m+k_{1} n\right)} \\
a^{\text {bound }}(m, n ; k) & =\mathrm{e}^{i u(m+n)+v(m-n)}, \quad u=\frac{k}{2}+\left(1-\frac{\Delta_{1}}{\left|\Delta_{1}\right|}\right) \frac{\pi}{2} .
\end{aligned}
$$


Here $C_{a b}=\cos \frac{k_{a}+k_{b}}{2}-\Delta_{1} \mathrm{e}^{i \frac{k_{a}-k_{b}}{2}}, \Delta_{1}=5 / 4-J_{\|} / J_{c}, v>0$ and

$$
\cos \frac{k}{2}=\left|\Delta_{1}\right| \mathrm{e}^{-v}
$$

The normalization factors,

$$
\begin{aligned}
Z_{\text {scatt }}\left(k_{1}, k_{2}\right) & =\sqrt{2} N \sqrt{\cos ^{2} \frac{k_{1}+k_{2}}{2}-2 \Delta_{1} \cos \frac{k_{1}+k_{2}}{2} \cos \frac{k_{1}-k_{2}}{2}+\Delta_{1}^{2}}, \\
Z_{\text {bound }}(k) & =\sqrt{\frac{N \cos ^{2} \frac{k}{2}}{\Delta_{1}^{2}-\cos ^{2} \frac{k}{2}}},
\end{aligned}
$$

depend on $N$ the number of rungs.

The corresponding dispersion laws are the following:

$$
\begin{aligned}
E^{\text {magn }}(k) & =J_{\perp}-\frac{3}{2} J_{c}+J_{c} \cos k \\
E^{\text {scatt }}\left(k_{1}, k_{2}\right) & =2 J_{\perp}-3 J_{c}+J_{c}\left(\cos k_{1}+\cos k_{2}\right), \\
E^{\text {bound }}(k) & =2 J_{\perp}+\left(\Delta_{1}-3\right) J_{c}+\frac{J_{c}}{\Delta_{1}} \cos ^{2} \frac{k}{2}
\end{aligned}
$$

As it follows from (25) the one-magnon gap $E_{\text {gap }}^{\text {magn }}$ and the one-magnon zone width $\Delta E^{\text {magn }}$ are given by the following formulas:

$$
E_{\text {gap }}^{\text {magn }}=J_{\perp}-\frac{3}{2} J_{c}-\left|J_{c}\right|, \quad \Delta E^{\text {magn }}=2\left|J_{c}\right| .
$$

\section{First order DSF for $\hat{H}^{s-r}+\hat{H}^{a s y m m}$}

From (4) and (20) follows that,

$$
\begin{aligned}
H_{n, n+1}^{\text {asymm }} \varepsilon_{\alpha \beta \gamma}|1\rangle_{n}^{\beta}|1\rangle_{n+1}^{\gamma} & =-i\left(J_{\|}^{\text {asymm }}-J_{\text {frust }}^{\text {asymm }}\right)|1\rangle_{n}^{\alpha}|0\rangle_{n+1} \\
& +i\left(J_{\|}^{\text {asymm }}+J_{\text {frust }}^{\text {asymm }}\right)|0\rangle_{n}|1\rangle_{n+1}^{\alpha},
\end{aligned}
$$

so,

$$
\begin{aligned}
{ }_{0}^{\alpha}\left\langle q, \text { magn }\left|\hat{H}^{\text {asymm }}\right| k_{1}, k_{2}, \text { scatt }\right\rangle_{0}^{\beta} & =\frac{2 \sqrt{2} J^{\text {asymm }}(q) \cos \frac{q}{2} \sin \frac{k_{1}-k_{2}}{2} \delta_{k_{1}+k_{2} q} \delta_{\alpha \beta}}{\left.\sqrt{N\left(\cos ^{2} \frac{q}{2}-2 \Delta_{1} \cos \frac{q}{2} \cos \frac{k_{1}-k_{2}}{2}+\Delta_{1}^{2}\right.}\right)}, \\
{ }_{0}^{\alpha}\left\langle q, \text { magn }\left|\hat{H}^{\text {asymm }}\right| k, \text { bound }\right\rangle_{0}^{\beta} & =-2 i \bar{J}^{\text {asymm }}(q) \frac{\sqrt{\Delta_{1}^{2}-\cos ^{2} \frac{q}{2}}}{\Delta_{1}} \delta_{k q} \delta_{\alpha \beta},
\end{aligned}
$$


where

$$
J^{\text {asymm }}(q)=J_{\text {frust }}^{\text {asymm }} \cos \frac{q}{2}-i J_{\|}^{\text {asymm }} \sin \frac{q}{2} .
$$

Considering $\hat{H}^{a s y m m}$ as a small perturbation we may obtain the corresponding corrections for the one- and two-magnon states. In the simplest case when the one-magnon mode does not intersect the two-magnon sector all the first order corrections to oneand two-magnon dispersions vanish. First order one-magnon contributions to the $S=1$ two-magnon states are the following:

$$
\begin{aligned}
&\left.\mid k_{1}, k_{2}, \text { scatt }\right\rangle_{1}= \frac{2 \sqrt{2} J^{\text {asymm }}\left(k_{1}+k_{2}\right) \cos \frac{k_{1}+k_{2}}{2} \sin \frac{k_{1}-k_{2}}{2}}{\sqrt{N\left(\cos ^{2} \frac{k_{1}+k_{2}}{2}-2 \Delta_{1} \cos \frac{k_{1}+k_{2}}{2} \cos \frac{k_{1}-k_{2}}{2}+\Delta_{1}^{2}\right)}} \\
&\left.\cdot \frac{1}{\left(E^{\text {scatt }}\left(k_{1}, k_{2}\right)-E^{\text {magn }}\left(k_{1}+k_{2}\right)\right)} \mid k_{1}+k_{2}, \text { magn }\right\rangle_{0}, \\
&\left.\mid k, \text { bound }\rangle_{1}=-2 i \frac{\sqrt{\Delta_{1}^{2}-\cos ^{2} \frac{k}{2}} \bar{J}^{\text {asymm }}(k)}{\Delta_{1}\left(E^{\text {bound }}(k)-E^{\text {magn }}(k)\right)} \mid k, \text { magn }\right\rangle_{0} .
\end{aligned}
$$

We use the following expression for the zero-temperature dynamical structure factor [1], [5], 6],

$$
S_{\alpha \beta}(\mathbf{q}, \omega)=\lim _{N \rightarrow \infty} \frac{1}{N} \sum_{\mu}\left\langle 0\left|\hat{\mathbf{S}}^{\alpha}(\mathbf{q})\right| \mu\right\rangle\left\langle\mu\left|\hat{\mathbf{S}}^{\beta}(-\mathbf{q})\right| 0\right\rangle \delta\left(\omega-E_{\mu}\right),
$$

where $\hat{\mathbf{S}}(\mathbf{q})$ is the Fourier transformation of spin associated with the two dimensional vector $\mathbf{q}=\left(q, q_{\text {rung }}\right)$. Here $q$ and $q_{\text {rung }}$ are the corresponding leg and rung components. Since the latter has only two possible values 0 and $\pi$ we may study them separately,

$$
\hat{\mathbf{S}}(q, 0)=\sum_{n} \mathrm{e}^{-i q n}\left(\mathbf{S}_{1, n}+\mathbf{S}_{2, n}\right), \quad \hat{\mathbf{S}}(q, \pi)=\sum_{n} \mathrm{e}^{-i q n}\left(\mathbf{S}_{1, n}-\mathbf{S}_{2, n}\right) .
$$

According to the following two formulas,

$$
[\hat{Q}, \hat{\mathbf{S}}(q, 0)]=0, \quad\{\hat{Q}, \hat{\mathbf{S}}(q, \pi)\}=\hat{\mathbf{S}}(q, \pi)
$$

we may reduce the matrix elements in (33)

$$
\langle\mu|\hat{\mathbf{S}}(q, 0)| 0\rangle=0, \quad\langle\mu|\hat{\mathbf{S}}(q, \pi)| 0\rangle=\sum_{\nu \in \mathcal{H}^{1}}\langle\mu \mid \nu\rangle\langle\nu|\hat{\mathbf{S}}(q, \pi)| 0\rangle,
$$

so $S_{\alpha \beta}(q, 0, \omega)=0$. For calculation of $S_{\alpha \beta}(q, \pi, \omega)$ let us notice that from (20) and (21) follows that

$$
\left\langle 0\left|\hat{\mathbf{S}}^{\alpha}(q, \pi)\right| k, \operatorname{magn}\right\rangle_{0}^{\beta}=\sqrt{N} \delta_{\alpha \beta} \delta_{k q}
$$


so, the DSF has purely diagonal form, $S_{\alpha \beta}(q, \pi, \omega)=\delta_{\alpha \beta} S(q, \pi, \omega)$, where

$$
S(q, \pi, \omega)=\sum_{\mu}\left|\langle\mu \mid q, \operatorname{magn}\rangle_{0}^{3}\right|^{2} \delta\left(\omega-E_{\mu}\right) .
$$

The unperturbed DSF corresponding only to $\hat{H}^{s-r}$ consists on a single one-magnon coherent peak

$$
S^{(0)}(q, \pi, \omega)=\delta\left(\omega-E^{\text {magn }}(q)\right) .
$$

In the first order with respect to the asymmetry we have to take into account only the two-magnon contributions. Using the substitution $2 \pi \sum_{k} \rightarrow N \int_{-\pi}^{\pi} d k$ we obtain the following formula:

$$
S^{(1)}(q, \pi, \omega)=A_{\text {bound }}(q) \delta\left(\omega-E^{\text {bound }}(q)\right)+A_{\text {scatt }}(q, \omega),
$$

where

$$
\begin{aligned}
A_{\text {bound }}(q) & =\frac{4\left|J^{\text {asymm }}(q)\right|^{2}\left(\Delta_{1}^{2}-\cos ^{2} \frac{q}{2}\right)}{\Delta_{1}^{2}\left(E^{\text {magn }}(q)-E^{\text {bound }}(q)\right)^{2}} \\
A_{\text {scatt }}(q, \omega) & =\frac{4\left|J^{\text {asymm }}(q)\right|^{2}\left(\cos ^{2} \frac{q}{2}-x^{2}(\omega)\right) \Theta\left(1-x^{2}(\omega)\right)}{\pi\left(\cos ^{2} \frac{q}{2}-2 \Delta_{1} x(\omega)+\Delta_{1}^{2}\right)\left(\omega-E^{\text {magn }}(q)\right)^{2}} .
\end{aligned}
$$

Here $\Theta(x)$ is the step function and $x(\omega)=\left(\omega-2 J_{\perp}+3 J_{c}\right) /\left(2 J_{c}\right)$.

The first term in (40) corresponds to the second coherent peak carried from the sector $\mathcal{H}^{\text {even }}$.

The formulas (41) and (42) will be correct only when the energy of the one-magnon mode is smaller than the energy of the bound state and the lower bound of the twomagnon continuum. Contrary due to the asymmetry mixing between $\mathcal{H}^{1}$ and $\mathcal{H}^{2}$ any intersection of the one- and two-magnon scattering sectors will result to magnon decay [10]. In order to avoid this possibility we shall obtain the "non-intersection" condition.

According to (25) and (26),

$$
2 J_{\perp}-3 J_{c}-2\left|J_{c}\right| \cos \frac{k_{1}+k_{2}}{2} \leq E^{s c a t t}\left(k_{1}, k_{2}\right) \leq 2 J_{\perp}-3 J_{c}+2\left|J_{c}\right| \cos \frac{k_{1}+k_{2}}{2},
$$

and the condition $E^{\text {magn }}\left(k_{1}+k_{2}\right)<E^{\text {scatt }}\left(k_{1}, k_{2}\right)$ reduces to the following form, $J_{c}(\cos k / 2+$ $\left.\left|J_{c}\right| /\left(2 J_{c}\right)\right)^{2}<J_{\perp} / 2$. This inequality will be automatically satisfied for $J_{c}<0$, while for $J_{c}>0$ it results to $2 J_{\perp}>9 J_{c}$ or using (28) to an equivalent form,

$$
E_{\text {gap }}^{\text {magn }}>\Delta E^{\text {magn }}
$$

The last formula has a clear interpretation. Really $E_{g a p}^{\text {magn }}-\Delta E^{\text {magn }}$ measures the difference between the one- and two- magnon sectors. When it is satisfied these sectors do not intersect a magnon decay is impossible and the formula (42) is correct. 


\section{Overview of experimental data for $\mathrm{CuHpCl}$}

As it was suggested in [5] the compound $\mathrm{CuHpCl}$ corresponds to the case $J_{\|}^{a s y m m}=0$, $J_{\text {frust }}^{\text {asymm }}=J_{\text {frust }}$. In other words it may be described by the Hamiltonian

$$
H_{n, n+1}^{\mathrm{CuHpCl}}=H_{n, n+1}^{\text {rung }}+H_{n, n+1}^{\text {leg }}+H_{n, n+1}^{\text {diag }}+H_{n, n+1}^{c y c},
$$

where the terms $H_{n, n+1}^{\text {rung }}, H_{n, n+1}^{\text {leg }}$ and $H_{n, n+1}^{c y c}$ are given by (3) and

$$
H_{n, n+1}^{\text {diag }}=J_{\text {diag }} \mathbf{S}_{1, n} \mathbf{S}_{2, n+1} .
$$

Here $J_{\text {diag }}=J_{\text {frust }}+J_{\text {frust }}^{\text {asymm }}=2 J_{\text {frust }}$.

If one suggests that the state (10) is the exact ground state then the condition (8) reduces to

$$
2 J_{\text {diag }}=J_{\|}-\frac{1}{2} J_{c}
$$

Under this condition (however not proved experimentally!) it is possible to estimate the parameters $J_{\perp}$ and $J_{c}$ from the formulas (25), (28) and experimental data. As it was presented in [11] $E_{\text {gap }}^{\text {magn }} \approx 10.8 \mathrm{~K}$, corresponds to $k=\pi$, however as it was shown in [12] by $k=0$ ESR measurements $E_{g a p}+2 \Delta E^{\text {magn }}=13.1 \mathrm{~K}$. This data agrees with the neutron scattering experiments [5], 6]. From (25) and (28) follows that $J_{c} \approx 1.2 \mathrm{~K}$ and $J_{\perp} \approx 13.8 \mathrm{~K}$.

Unfortunately any quantitative interpretation fails for the neutron scattering data obtained in [6]. Really all the scans presented here correspond to the scattering with $q_{\text {rung }}=0$. However as it was shown in the previous section $S_{\alpha \beta}(q, 0, \omega)=0$. Therefore appearance of the scattering peaks in Figs 9 and 10(a) of the Ref. 6 may be explained only by a deviation of the initial state of the ladder from the singlet-rung vacuum (10). The strength of this deviation may be estimated only by comparison the data presented in [6] with the same one related to the scattering with $q_{\text {rung }}=\pi$. However the latter is not yet obtained.

In despite of the quantitative disagreement at $q_{\text {rung }}=0$ our argumentation qualitatively confirms the appearance of the second coherent peak in the structure factor.

\section{Summary}

In this paper we have demonstrated the principal difference between the excitation spectrums of symmetric and asymmetric spin ladders. For the symmetric one the Hilbert 
space splits on two invariant subspaces $\mathcal{H}^{\text {even }}$ and $\mathcal{H}^{\text {odd }}$. In this case only the sector $\mathcal{H}^{\text {odd }}$ gives a nonzero contribution to the dynamical structure factor. However the picture is quite different for an asymmetric spin ladder. The asymmetry term mixes both the subspaces and the two-magnon bound state from $\mathcal{H}^{\text {even }}$ contribute to the DSF resulting to the appearance of the second coherent peak.

As an illustration we have obtained the first order DSF for the special model of asymmetric spin-ladder with exact singlet rung ground state. The suggested model was applied to the probably asymmetric spin-ladder compound $\mathrm{CuHpCl}$ for which the existence of the second coherent peak was observed experimentally. Despite the full agreement between our special model and the experimental data was not confirmed some of the interaction constants were estimated from the inelastic neutron scattering and ESR data.

The authors are very grateful to S. V. Maleev for helpful discussions.

\section{References}

[1] S. W. Lovesey, Theory of Neutron Scattering from Condensed Matter (Clarendon, Oxford, 1984)

[2] T. Barnes, E. Dagotto, J. Riera, E. S. Swanson, Phys. Rev. B 47, 3196 (1993)

[3] A. A. Nerseysan and A. M. Tsvelik, Phys. Rev. Lett. 78, 3939 (1997)

[4] B. Chari, O. Piovesana, T. Tarantelli, and P. F.Zanazzi, Inorg. Chem. 29, 1172 (1990)

[5] P. R. Hammar, D. H. Reich, C. Broholm, F. Trouw, Phys. Rev. B, 57, 7846 (1998)

[6] M. B. Stone et al, Phys. Rev. B, 65, 064423 (2002)

[7] A. K. Kolezhuk and H.-J. Mikeska, Int. J. Mod. Phys. B 12, 2325 (1998)

[8] P. N. Bibikov, Phys. Rev. B 72012416 (2005)

[9] V. N. Kotov, O. P. Sushkov, R. Eder, Phys. Rev. B 59, 6266 (1999)

[10] M. E. Zhitomirsky, Phys. Rev. B 73, 100404(R) (2006)

[11] G. Chaboussant, P. A. Crowell, L. P. Lévy, O. Piovesana, A. Madouri, D. Mailly, Phys. Rev. B 55, 3046 (1997) 
[12] M. Hagiwara, Y. Narumi, K. Kindo, T. Nishida, M. Kaburagi, T. Tonegawa, Physica B 246-247, 234 (1998) 